\title{
CURRENT IDEAS ABOUT THE PRODUCTION OF THE ELECTROGLOW OF URANUS
}

\author{
J. T. Clarke
}

Department of Atmospheric, Oceanic and Space Sciences, The University of Michigan, Ann Arbor, MI 48109-2143, U.S.A.

ABSTRACT

A process known as "electroglow" was identified by Broadfoot et al. /1/ shortly after the Voyager Uranus encounter as soft charged particle excitation of atmospheric gas in the presence of sunlight and in excess of the available photoelectron energy. Localized aurora and diffuse $\mathrm{H}$ and $\mathrm{H}_{2}$ band emissions have been observed from Uranus, and the $\mathrm{H}$ Ly $\alpha$ is highly variable. A high upper atmospheric temperature of $800 \mathrm{~K}$ and enhanced ionization layers were also observed at Uranus, and these phenomena are all similar to (but more pronounced than) those observed at Jupiter and Saturn. It is clear that the upper atmospheres of the outer planets are driven by more than solar EUV radiation. A theory has been advanced for the energization of photoelectrons and ions by ionospheric fields produced by the dynamo action of the atmosphere, and the potential contribution of scattering processes to these emissions is being re- analyzed.

\section{INTRODUCTION}

Following the Voyager encounters with Jupiter and Saturn, there were reports of UV emissions which did not exhibit the properties expected from direct scattering of sunlight. For example, on Jupiter there is a localized region of bright equatorial $\mathrm{H}$ Ly $\alpha$ emission which rotates in the rest frame of the magnetic field, rather than with the atmosphere $/ 2,3 /$. On Jupiter there also appears a brightening of the $\mathrm{Ly} \alpha$ and the $\mathrm{H}_{2}$ bands at the dawn terminator, and several $\mathrm{kR}$ of $\mathrm{Ly} \alpha$ emission extends up to $20^{\circ}$ into the "dark" atmosphere /4/. Diffuse $\mathrm{H}_{2}$ band emissions on Jupiter and Saturn were interpreted as being produced by soft $(50 \mathrm{eV})$ electron excitation, although it appeared that the energy required to produce these emissions is greater than the available energy for excitation by photoelectrons $/ 5 /$. It was observed that this emission appeared predominantly in the sunlit atmospheres, and decreased greatly in the shadow of Saturn's rings. The altitude profiles of the Ly $\alpha$ and $\mathrm{H}_{2}$ emissions from Saturn peak at relatively high atmospheric densities and the emissions are limb brightened, suggesting an optically thin emission process. Following more detailed analyses of the Voyager Ultraviolet Spectrometer (UVS) spectra of Jupiter /6/ and Saturn $/ \pi /$, it was concluded that these diffuse emissions are largely restricted to the sunlit atmosphere, and that they are produced by soft $(10-50 \mathrm{eV})$ electron and proton excitation with a peak near or just above the homopause in altitude and extending through the higher atmosphere.

\section{OBSERVATIONS}

Observations by the International Ultraviolet Explorer (IUE) /8/ and then by the UVS /1/ of an unexpectedly high brightness in these emissions from Uranus re-emphasized the important clue that the radiated energy is greater than the solar EUV (i.e. plotoelectron) energy input into the atmosphere. In fact, the ratio of UV emission to solar input is substantially higher on Uranus than either Jupiter or Saturn (see Table 1). Although photoelectrons may act as a trigger for the process (which decays rapidly in the absence of sunlight), the energy generation appears to occur locally in the atmosphere since there is no observed 
Table 1

\begin{tabular}{|c|c|c|c|c|}
\hline Planet & Day/Year & Solar EUV Flux ${ }^{2}$ & Emission Flux ${ }^{3}$ & Emission $/$ Solar $^{2}$ \\
\hline Jupiter & $190 / 1979$ & $\overline{0.14 \mathrm{ergs} / \mathrm{cm}^{2}-\mathrm{sec}}$ & $.07-.13 \mathrm{ergs} / \mathrm{cm}^{2}-\mathrm{sec}$ & $3-6$ \\
\hline Saturn & $238 / 1981$ & .056 & $.022-.048$ & $3-6$ \\
\hline Uranus & $024 / 1986$ & .0036 & $.014-.045$ & $25-80$ \\
\hline
\end{tabular}

${ }^{1}$ Solar EUV fluxes at each planet are based on data of Hinteregger et al. (1981) scaled to day of Voyager encounter by solar 10.7 cm flux (W. Hoegy 1987, pers. comm.)

2Observed electroglow emission flux divided by photoelectron excitation (assumed to be Solar ECV Flux $\times 15 \%$ )

${ }^{3}$ The estimates of particle-excited emission assume the following:

$\begin{array}{lccc} & \underline{H L Y \alpha} & \mathrm{H}_{2} & \text { Total } \\ \text { Jupiter } & 3-5 \mathrm{kR} & 1.4-3.0 \mathrm{kR} & 4.4-8.0 \mathrm{kR} \\ \text { Saturn } & 1-2 \mathrm{kR} & 0.35-1.0 \mathrm{kR} & 1.35-3.0 \mathrm{kR} \\ \text { Uranus } & 0.5-1.0 \mathrm{kR} & 0.40-1.8 \mathrm{kR} & 0.9-2.8 \mathrm{kR}\end{array}$

correlation between the emission and precipitating magnetospheric charged particles. The process producing both Ly $\alpha$ and $\mathrm{H}_{2}$ emissions was judged to be sufficiently unique to be named "electroglow" by Broadfoot et al. $/ 1 /$.

The sunlit disk average Ly $\alpha$ brightness of Uranus has ranged from 800-2400 R over 4 years of IUE observations (which includes the auroral emission, Figure 1 from /8/), and was measured at $1500 \mathrm{R}$ by the UVS during the Voyager encounter (not including auroral emission /1/). The variability of the Ly $\alpha$ emission is independent of the slow decrease in solar Ly $\alpha$ flux toward solar minimum $/ 8 /$. Allowing for $30 \%$ absorption by interplanetary $\mathbf{H}$ atoms between Uranus and the Earth, and a $20 \%$ calibration difference between the UVS and IUE, the 1500 R UVS measurement (near Uranus) corresponds to $800 \mathrm{R}$ as measured by ILE (from Earth orbit). This is near the minimum brightness observed over four years by IUE, and is consistent with the full level of solar-scattered emission plus a minimum amount of auroral and electroglow emissions at that time.

Over the course of the IUE observations the emission varied from $800-2400 \mathrm{R}$, with variations of a factor of 2 in as little as six hours. The UVS also detected up to $50 \%$ variations in the emissions on the approach to Uranus /1/. These observations are somewhat uncertain due to an uncertainty in spacecraft pointing, but at a minimum the ratio of $\mathrm{Ly} \alpha / \mathrm{H}_{2}$ was changing with time (D. Shemansky, pers. comm.). Neither the UVS nor IUE can spatially distinguish the source of the variable emission, which could be either polar aurora or the electroglow. However, the unusually high solar wind pressure and low magnetospheric plasma densities during the Uranus encounter suggest that during closest approach there was a relaxation following a magnetic storm, consistent with a lack of bright aurora at that time. The fast variations in the IUE data may be more easily explained by localized auroral emission regions, which would relieve some of the requirements on total energy in the electroglow process. In addition, the tilted and offset magnetic field of Uranus provides an order of magnitude difference in field strength between sunlit and dark hemispheres $/ 9 /$, which means that charged particles that mirror in the atmosphere near the sunlit pole will mirror more than $1 \mathrm{R}_{U}$ above the atmosphere near the dark pole. Over a wide range of L-shells in the Uranian magnetosphere, the solid angle of the loss cone into the sunlit hemisphere is roughly 3-4 times larger than the loss cone into the dark atmosphere, which would result in a comparable ratio of fluxes of precipitating auroral particles for an isotropic distribution of plasma. The dayside aurora are therefore expected to be several times brighter than the nightside aurora. There may also be strong ionospheric currents produced by magnetospheric convection.

The diffuse Ly $\alpha$ emission from Uranus is comparable in brightness to the Ly $\alpha$ emission from Saturn, and it is difficult to separate the contributions from scattering processes and particle excitation. The dark hemisphere Ly $\alpha$ brightness is $170 \mathrm{R}$, which suggests that some of the emission is produced by charged particles. The subsolar $\mathrm{H}_{2}$ brightness is in the range $400-1800$ $R$, depending on the assumed relative contributions of the $\mathrm{H}_{2}$ band $\mathrm{a}-\mathrm{b}$ continuum and Rayleigh scattered sunlight, and no detection of diffuse $\mathrm{H}_{2}$ emission from the dark side has been reported, although without a specified upper limit /1/. The altitude distribution of the emissions is similar to Saturn, with the peak for both $\mathrm{Ly} \alpha$ and $\mathrm{H}_{2}$ bands above the homopause and decreasing with altitude up to the exosphere (Figure 2). It was initially suggested that the excitation energy at Uranus might be lower than at the other 2 planets (a Maxwellian distribution with a peak at $3 \mathrm{eV}, / 1 /$ ). However, more recent work suggests that the reported $\mathrm{H}_{2} \mathrm{a}-\mathrm{b}$ continuum may alternately be attributed largely to Rayleigh scattered sunlight, implying that the $\mathrm{H}_{2}$ excitation energy is actually closer to the $10-50 \mathrm{eV}$ range observed at Jupiter and Saturn $/ 10 /$.

There are other phenomena observed from the outer planet upper atmospheres that imply considerable energy deposition and which may be related to the electroglow emissions. For example, Jupiter, Saturn, and Uranus all have unexpectedly hot upper atmospheres (on the order of $1000 \mathrm{~K}$ ), and each planet's topside ionosphere is extended in altitude (generally consistent with 

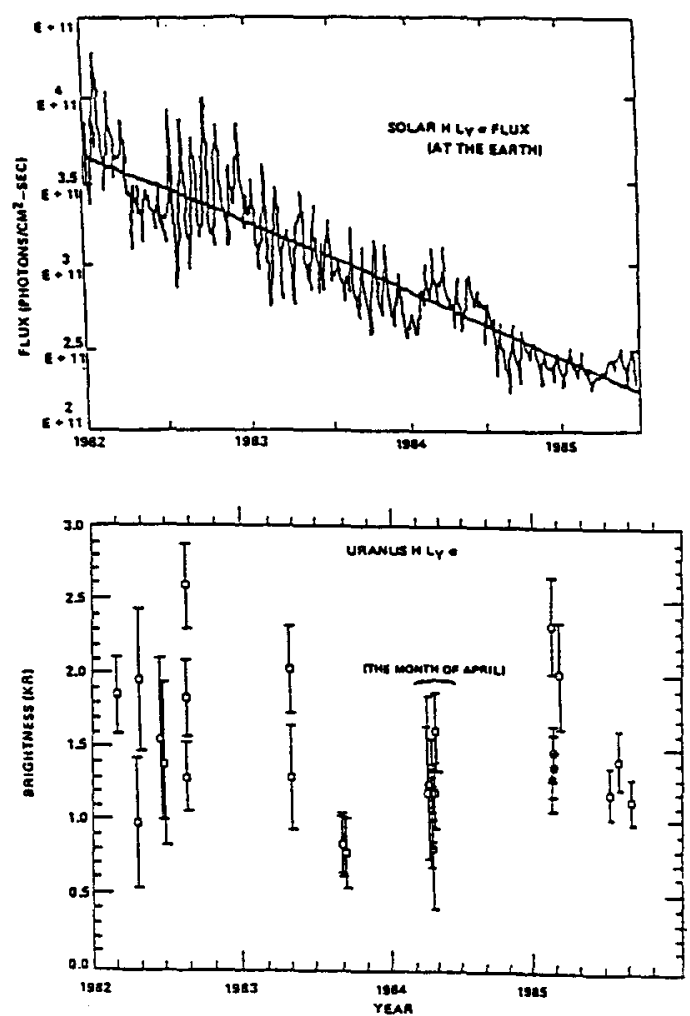

Fig. 1 Uranus H Ly $\alpha$ brightness measured by IUE and solar Lyman $\alpha$ flux measured by SME (from $/ 8 /$ ).

the observed high neutral temperature). Particularly at the distance of Uranus from the sun, the solar input is insufficient to provide this heating, and it appears that the exobase temperature is almost independent of solar flux. The observations of neutral hydrogen clouds in the inner magnetospheres of Saturn and Uranus are consistent with the measurements of extended corona, and several workers are now investigating whether superthermal processes are required to supply these neutral clouds. Velocity information about these extended corona would be invaluable in identifying the coronal supply mechanism.

\section{SCATTERING AND FLUORESCENCE OF SOLAR RADIATION}

Because of the apparent spatial correlation between much of the diffuse emission and incident sunlight, Yelle and collaborators /10/ have re-analyzed the potential contributions of resonant scattering, Rayleigh scattering, and $\mathrm{H}_{2}$ resonance fluorescence of solar UV radiation to the diffuse emission. These calculations apply to those emissions which obey a solar phase angle dependence, the correct spectral shape, and the temporal variations observed in the solar flux. These processes are thus not electroglow, since electroglow has been defined on the basis of observed phenomena which are either independent of or in excess of the available solar energy. For example, none of the emissions discussed in the introduction are explainable by scattering or fluorescence of solar radiation. However, the more diffuse emission which can be explained by solar scattering, the lower the energy requirements on the electroglow.

Yelle et al. / $10 /$ have shown that the diffuse $\mathrm{H}_{2}$ band emission measured by the Voyager UVS at wavelengths below $1100 \AA$ scales in brightness as $1 / \mathrm{R}^{2}$ from Jupiter to Saturn to Uranus (Figure 3). The emission below $1100 \AA$ represents roughly $1 / 3$ of the total $\mathrm{H}_{2}$ band emission. In addition, much of the diffuse $\mathrm{H}_{2}$ band emission appears to decrease with a roughly cosine 


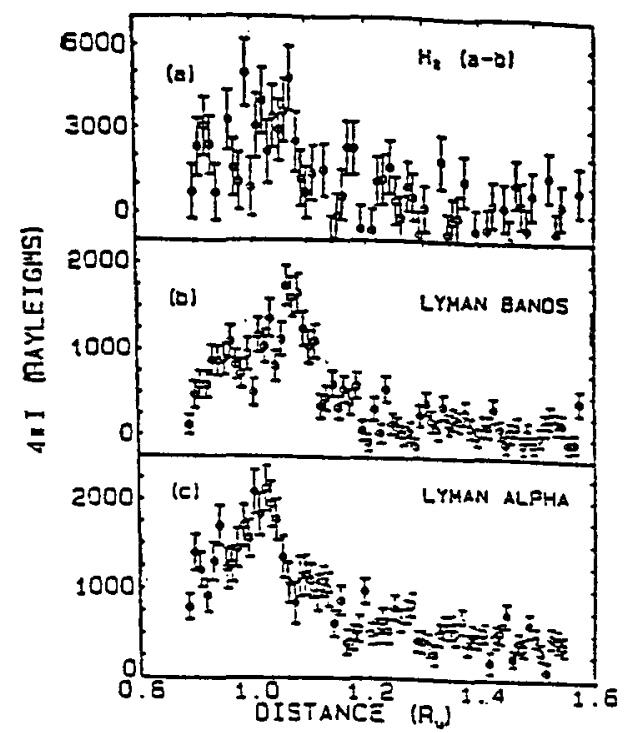

Fig. 2 Altitude profiles of Uranus UV emissions (from $/ 1 /$ ).

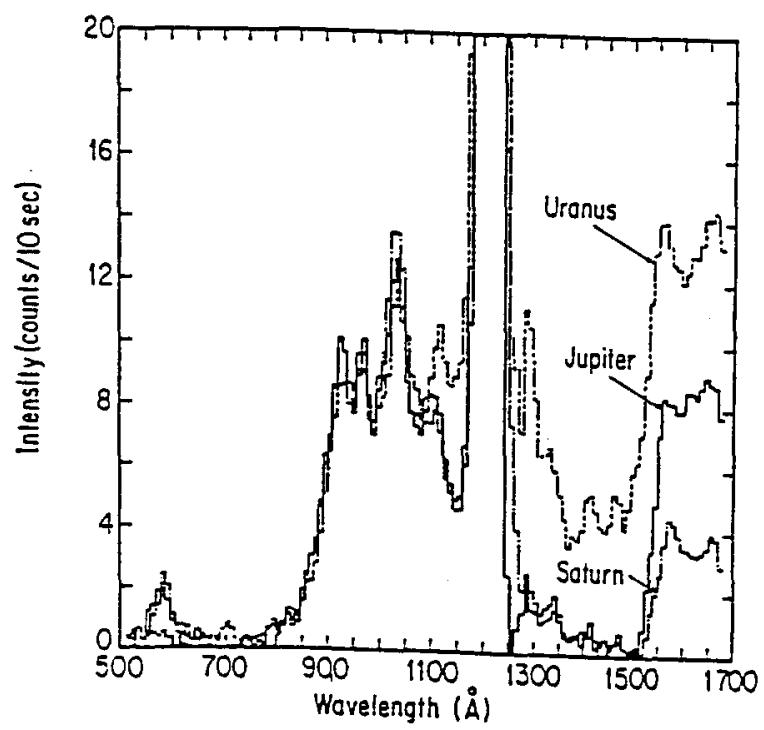

Fig. 3 Relative spectra of 3 outer planets scaled by $1 / R^{2}$ (from $/ 10 /$ ).

dependence on solar zenith angle. The authors suggest that the diffuse $\mathrm{H}_{2}$ bands from Uranus may be largely produced by resonance fluorescence of solar FUV emission (RF). The mechanism involves absorption of solar radiation by $\mathrm{H}_{2}$ in the ground vibrational state. These transitions become optically thick and therefore line broadened, so that the efficiency of RF therefore depends non-linearly on the optical depth of the $\mathrm{H}_{2}$ column above the absorbing hydrocarbons. $\mathrm{H}_{2}$ fluorescent emission may occur between upper vibrational states which are optically thin, so that optically thick absorption of the solar FUV radiation may result in the observed optically thin emission. Initial calculations of the fluorescent intensity at Jupiter suggest that roughly $1 / 2$ of the input solar spectrum will be re-radiated and predict that roughly $2 / 3$ of the observed $\mathbf{H}_{2}$ band emissions at Jupiter 
may result from this process $/ 10 \%$. However, the spectrum of RF at moderately high resolution is very different from that - of electron excitation, offering an eventual tool for discriminating between these processes. In the case of the Ly $\alpha$ emission, there are several potential excitation processes. The sum of the modeled brightnesses from all processes exceeds the observed brightness, so that the relative contributions of scattering and charged particle excitation must be inferred from spatial or temporal variations rather than from energetic arguments.

Several groups are now calculating the RF efficiency and spectrum. The upper atmosphere of Uranus is particularly clear of UV-absorbing hydrocarbons, compared with Jupiter and Saturn, yielding a high efficiency for RF. Presently all groups agree that there is a strong component of solar scattered emission in the Uranus UV spectrum, particularly in the FUV range. Most workers also conclude that there is very little $\mathrm{RF}$ emission at Saturn due to a thin column of $\mathrm{H}_{2}$. In addition, the magnitude and spectral shape of the solar EUV flux changed considerably between the Jupiter (solar max.) and Uranus (solar min.) encounters, so the observed $1 / R^{2}$ variation among the outer planets is not explained by $R F$. In principle the contribution of $H_{2}$ $\mathrm{RF}$ is calculable if the atmospheric structure and solar flux are known, so that further careful modeling may reveal the relative contribution of RF without additional observations. However, there is not yet general agreement on the relative contributions of solar scattering and electron excitation to the diffuse emission. There is also a pronounced need for further observations of the $\mathrm{H} \mathrm{Ly} \alpha$ and $\mathrm{H}_{2}$ band emissions from Uranus to establish the variability time scale(s) and resolve the many questions about spatial structure. Unfortunately only IUE disk average Ly $\alpha$ measurements are possible at this time.

\section{IN-SITU ACCELERATION}

Since there are UV emissions whose spatial/temporal distributions are unexplainable by solar scattering and/or fluorescence, and the energy requirements for UV emission and heating are much higher than the solar input on Uranus, other workers have pursued explanations for these phenomena. Since the night hemispheres of all the planets appear much darker than the sunlit hemispheres, the spatial and altitude distributions of the emissions generally match those expected for photoelectrons $/ 11 /$, and the low latitude UV emissions do not follow the expected correlations with precipitating magnetospheric charged particles, it is logical to investigate the in-situ acceleration of photoelectrons and ions. The possibility that electric fields might accelerate these particies contributed to the name "electroglow".

\section{IONOSPHERIC DYNAMO}

The application of terrestrial dynamo theory to the cases of the outer planets has been investigated by Clarke, Hudson, and Yung /12/. Strong electric currents in the $E$ and $F$ regions of the Earth's ionosphere are produced by an atmospheric dynamo, whose driving force stems from charge separation by the interaction of neutral winds with ionospheric plasma. A derivation and review of dynamo theory and its manifestations is given by Rishbeth and Garriott $/ 13 /$. If $\omega_{e}$ is the electron gyrofrequency and $\nu_{e n}$ is the collision frequency of electrons with neutrals, then in the altitude range where:

$$
\nu_{\text {en }} / \omega_{e} \ll 1 \text { and } \quad \nu_{\text {in }} / \omega_{i} \simeq 1
$$

the ions will be dragged along by a neutral wind while the electrons remain fixed on magnetic field lines, resulting in charge separation in the direction of the neutral wind at dawn/dusk boundaries where the winds reverse. On the Earth the peak dynamo currents are measured at altitudes near $110 \mathrm{~km}$ (somewhat higher than the altitude of maximum charge separation, ef. $/ 13 /$ ) and extend to much higher altitudes. The equivalent conditions on Uranus, Saturn, and Jupiter occur just above the homopause on each planet. Dynamos will operate in the outer planet atmospheres, subject to the neutral wind velocity, the strength of the magnetic field, and the charged particle density. Preliminary modeling of the Jovian dynamo has been presented /14/. The question is how much of the energy will go into FUV radiation, how much into Joule heating, etc.

Acceleration of the magnetized electrons requires field-aligned potentials. Collisional drag of protons at $100 \mathrm{~m} / \mathrm{sec}$ gives only 5 $\mathrm{x} 10^{-5} \mathrm{eV}$, which is not sufficient for excitation of UV emissions and suggests that the ions are also accelerated by field-aligned potentials. Field-aligned (Birkeland) currents in the Earth's $\mathrm{F}$ and $\mathrm{E}$ regions result from current closure along the bighly conductive magnetic field lines driven by $E$ and $F$ region polarization due to the dynamo action $/ 15 /$. The basic ionospheric circuit is shown in Figure 4 for mid-latitude and equatorial cases. The conductivity for electron flow along field lines is extremely high, and the Pederson $\left(\mathrm{j}_{E}\right)$ conductivity in the $\mathrm{E}$ region is also high and carried mainly by protons. Ion Pedersen conductivity provides cross field line current closure in both the $\mathrm{E}$ and $\mathrm{F}$ region legs of the circuit. The $\mathrm{E}$ region conductivity is greatly reduced at night by recombination, so dynamo driven Birkeland currents are larger during the day relative to a fixed neutral wind velocity $/ 15 \%$. Sufficiently high Birkeland currents can give rise to plasma instabilities which inhibit the bulk of the electron drif at the expense of accelerating high-energy electrons to superthermal energies, a phenomenon known as anomalous resistivity. A weaker version of the anomalous resistivity in the auroral zones acting at low and mid-latitudes may accelerate photoelectrons (and ions) above some critical energy along hundreds of kilometers parallel to $B$ in the ionosphere to produce the electroglow 

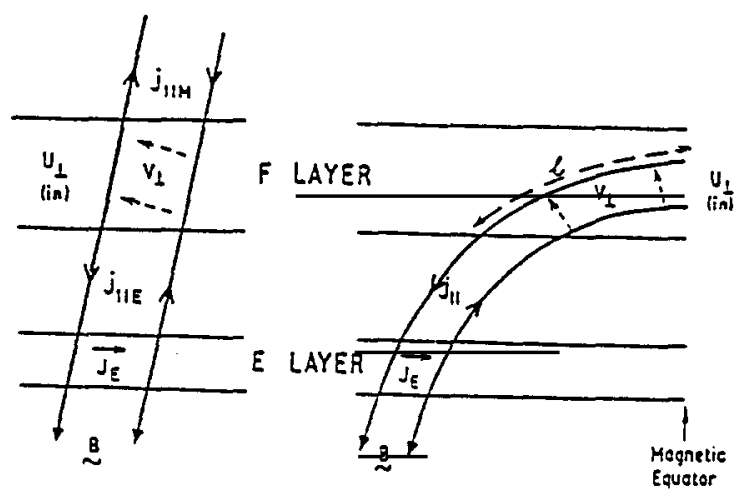

Fig. 4 Ionospheric circuits on the Earth (from /15/).

emissions. The exciting charged particles therefore represent a superthermal population in the ionosphere, since anomalous resistivity preferentially accelerates particles in the high energy portion of the distribution. It is the presence of Birkeland currents in the ionosphere, rather than direct dynamo action, that leads to UV emissions by the mechanism of anomalous resistivity. Any contributions to Birkeland currents from magnetospheric processes or from magnetic conjugate regions with relatively higher ionospheric densities may lead to locally enhanced UV emission.

\section{EXCITATION PROCESSES}

The fate of photoelectrons in a pure hydrogen atmosphere has been investigated by Cravens et al. /16/. Photoelectrons lose energy in collisions with $\mathrm{H}_{2}$ by electronic and vibrational excitation, ionization, and dissociation, and are eventually thermalized. Figure 5 a shows the overall loss function $L(E)$ for electrons in $\mathrm{H}_{2}$ and the contributions to $L(E)$ from the various processes. Given the initial energy of an electron, the subsequent energy cascade $/ 17 /$ can be described by the equation:

$$
\frac{d E}{d x}=\alpha-n L(E)
$$

where $d E / d x$ is the rate of energy loss per unit length $(\mathrm{eV} / \mathrm{cm}), n=$ number density of ambient gas (molecules $\mathrm{cm}^{-3}$ ), and $L(E)$ is the energy loss function $\left(\mathrm{cm}^{2} \mathrm{eV}\right)$. An electric force $\alpha(\mathrm{eV} / \mathrm{cm})$ may also be acting on the photoelectrons. Since $L(E)$ and $n$ are always positive, the second term describes a monotonic downward cascade of energy. However, assuming that $\alpha>0$, there may be an increasing electron energy if successive paths are co-aligned (i.e. the electron scattering is forward-peaked). The mean electron energy increases if:

$$
\alpha>n L(E)
$$

At $\mathrm{n}=10^{12} \mathrm{~cm}^{-3}$ and electron energy up to $\mathrm{E}=10 \mathrm{eV}$, a local electric field of $2 \mathrm{mV} / \mathrm{m}$ is sufficient to compensate for the collisional loss processes so that the electron energy will be maintained.

At an energy of roughly $10-15 \mathrm{eV}$ a steep increase in $\mathrm{L}(\mathrm{E})$ is encountered, representing cross section increases for dissociation, ionization, and excitation of the Lyman and Werner band systems of $\mathrm{H}_{2}$ (Figure $5 \mathrm{~b}, / 18 /$ ). Near $10 \mathrm{eV}$ the threshold for $\mathrm{B}_{2}$ dissociation is reached, and just above $11 \mathrm{eV}$ is the threshold for resonant excitation of excited state $\mathrm{H}_{2}^{-}$leading to Lyman band emission (J. Ajello, pers. comm.). This process in particular produces vibrational features observed in the UVS spectra of Uranus that are not produced by direct excitation of the B state. In addition, the cross section for elastic seattering is decreasing toward higher energies, and will be exceeded by the combined (ionization + excitation) cross section at roughly $40-50 \mathrm{eV}$. At this energy an electron will be more likely to directly excite or ionize an $\mathrm{H}_{2}$ molecule with each collision, and it thus represents a practical upper limit to the energy an electron will achieve before losing 10-15 eV to one of these processes. This is the $\mathrm{H}_{2}$ equivalent of the classic Franck-Hertz experiment, which demonstrated the existence of quantum levels in atoms through a decrease in current and the initiation of $2537 \mathrm{~A}$ radiation from $\mathrm{Hg}$ gas at the $4.9 \mathrm{eV}$ potential of the first excited state /19/. This phenomenon does not occur with the same magnitude in the Earth's atmosphere, because the elastic scattering cross sections for $\mathrm{N}_{2}$ and $\mathrm{O}_{2}$ remain several times greater than those for excitation at all energies. In an $\mathrm{H}_{2}$ atmosphere relatively more of the 


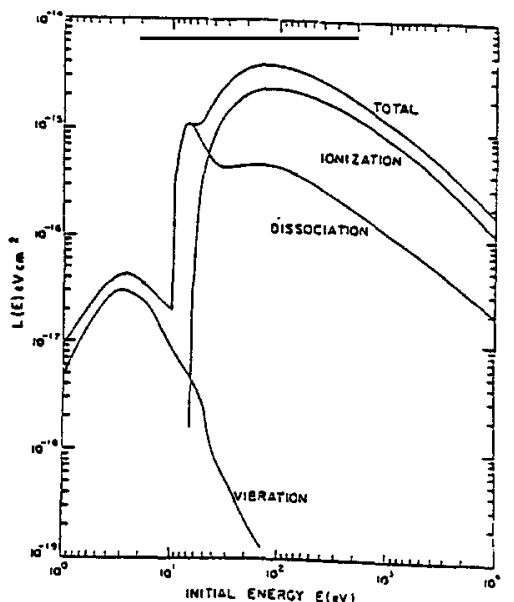

Fig. 5a Energy loss function for electron impact on $\mathrm{H}_{2}$ (from /16/).

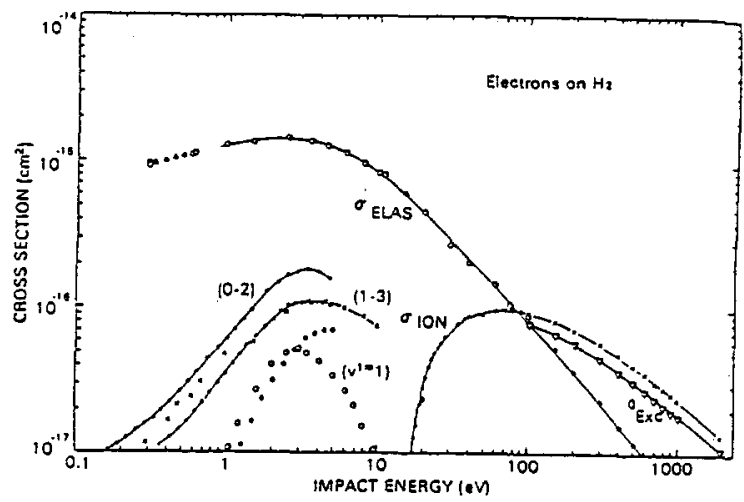

Fig. 5b Various cross sections for electron impact on $\mathrm{H}_{2}$ (from /18/).

energy from linearly accelerated electrons is dissipated as radiation and relatively less as Joule heating, and the $\mathrm{H}_{2}$ electroglow will exhibit a spectrum characterized by $10-50 \mathrm{eV}$ excitation.

The calculation of the generation of field-aligned potentials by neutral winds via anomalous resistivity is discussed by Hudson, Clarke, and Warren /20/. They show that for Uranus, assuming the maximum wind velocity of $200 \mathrm{~m} / \mathrm{sec}$ measured at the cloud tops with respect to the magnetic field $/ 21 /$, total energies in excess of collisional losses of $10-20 \mathrm{eV}$ may be gained by photoelectrons and ions over reasonable path lengths in the ionosphere. The next step will be to perform a more detailed calculation of the angle-dependent effects of elastic scattering on the acceleration and collisional energy loss. 
IONOSPHERIC DISTRIBUTION

The brightness of electroglow emission produced by ionospheric currents will depend on the photoelectron and ion densities and energy spectra, on the direction and magnitude of the neutral wind, on the dip angle of the magnetic field, and on the contribution to Birkeland currents from magnetospheric convection. The brightness distribution will also depend on global circulation patterns of both the neutral atmosphere and ionospheric plasma. Thermospheric winds are driven in part by nearUV solar heating of the lower atmosphere and in part by in situ FUV absorption. Since the near-UV solar flux is relatively constant (both short-term and long-term), this component will not vary greatly with time. Auroral heating will also contribute to thermospheric winds at high latitudes and may dominate the energetics over much of the atmosphere, at least on Jupiter. The auroral component will, of course, be much more variable than the solar component.

The sum of these components will determine the global neutral wind patterns, and their estimation will be an important step in evaluating the potential for the dynamo mechanism to produce the electroglow. In view of the large sizes, rapid rotation rates, intense auroral emissions, and decreased solar input at the outer planets, their thermospheric wind patterns should be very different from those on the Earth. Unfortunately, there is presently no observational data on the thermospheric winds from any outer planet. The peculiar magnetic geometries, especially on Uranus, will also complicate the generation of dynamo electric fields. In particular, the extended regions on Jupiter and Uranus of nearly horizontal magnetic field are conducive to the generation of extended regions of tropical arc H Ly $\alpha$ emission. The position of Jupiter's Ly $\alpha$ bulge region is consistent with the most extended region of horizontal magnetic field along Jupiter's equator, and preliminary modeling of the surface field of Uranus indicates an even more extended region of nearly horizontal magnetic field on the presently illuminated hemisphere 19/. An important plasma transport process on Uranus will be conjugate heating, and the degree of plasma flow from sunlit to night hemispheres will be particularly great as a result of the $60^{\circ}$ tilt of the magnetic field (Figure 6 ). Conjugate heating may aiso explain the dawn crescent UV emissions on Jupiter. This effect will produce relatively depleted ionospheric densities near the terminator in the sunlit atmosphere, and relatively enhanced densities in the conjugate night atmosphere. If the same effect occurs on Jupiter and Uranus, emissions similar to Jupiter's dawn crescent might be observed near the Uranus terminator.

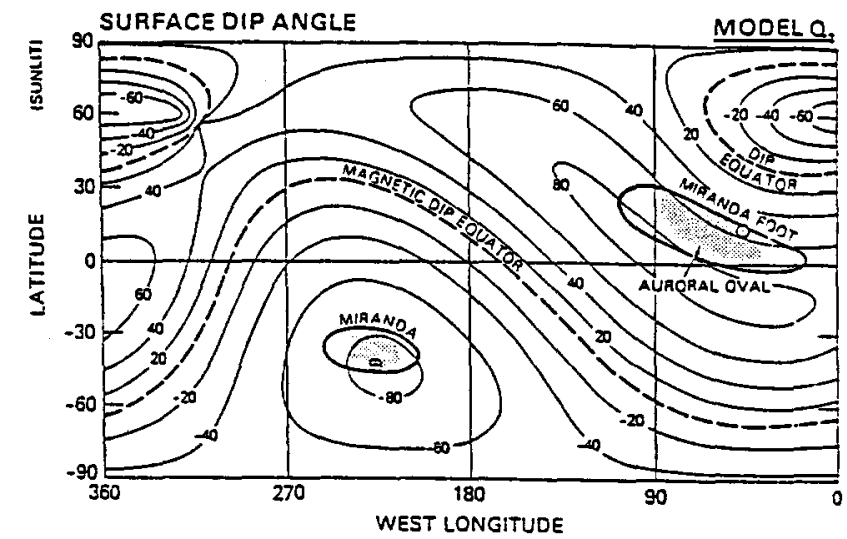

Fig. 6 The surface magnetic field of Uranus (from $/ 9 /$ ).

\section{ATMOSPHERIC HEATING}

It is also clear from Figure $5 \mathrm{~b}$ that electrons accelerated in the range $1-6 \mathrm{eV}$ will preferentially lose their energy in the excitation of vibrational transitions of $\mathrm{H}_{2}$, which will contribute to heating the local neutral atmosphere. Since roughly 1 in 5 collisions results in vibrational excitation at $3 \mathrm{eV}$ and elastic scattering is largely isotropic at the lower energies, particles below roughly 5 $\mathrm{eV}$ contribute to heating with a high efficiency. The ratio of heating/UV excitation in the electroglow process then depends on the lower cut-off energy above which anomalous resistivity will accelerate electrons (i.e. the lower the cut-off, the more heating compared to UV radiation). The vibrational excitation is potentially capable of heating the upper atmospheres of all 3 planets to the observed temperatures of $400-1200 \mathrm{~K}$, although the magnitude of this contribution compared to other heating processes (such as inertial gravity waves) has yet to be established. 


\section{CONCLUSION}

It appears that several different processes contribute to the emissions that were initially classified as electroglow. Much of the variable H Ly $\alpha$ emission from Uranus may be produced by localized aurora, as originally interpreted. A significant fraction of the diffuse emission from the sunlit atmosphere of Uranus appears to be due to solar scattering and $\mathrm{H}_{2}$ resonance fluorescence. There are also low latitude emissions from Uranus, Saturn, and Jupiter that are not explained by solar scattering, for example with spatial structure independent of the solar flux. These emissions appear to be explainable in the context of ionospheric acceleration processes that have been studied in more detail on the Earth, but applied to an $\mathrm{H}_{2}$ atmosphere. Observations with better angular and more continuous time resolutions will be important in identifying the specific emission processes

\section{REFERENCES}

1. A.L. Broadfoot et al., Ultraviolet Spectrometer Observations of Uranus, Science, 233, 74, (1986)

2. A.J. Dessler, B.R. Sandel, and S.K. Atreya, The Jovian Hydrogen Bulge: Evidence for Co-rotating Magnetospheric Convection, Planet. Soace Sci., 29, 215, (1981)

3. J.T. Clarke, H.W. Moos, and P.D. Feldman, IUE Monitoring of the Spatial Distribution of the H Ly $\alpha$ Emission from Jupiter, Astroph. I. Lett., 245, L127, (1981)

4. J.C. McConnell, B.R. Sandel, and A.L. Broadfoot, Airglow from Jupiter's Nightside and Crescent: Ultraviolet Spectrometer Observations from Voyager 2, Lcarus, 43, 128, (1980)

5. A.L. Broadfoot et al., Extreme Ultraviolet Observations from Voyager 1 Encounter with Saturn, Science, 212, 206, (1981)

6. D.E. Shemansky, An Explanation for the H Ly $\alpha$ Longitudinal Asymmetry in the Equatorial Spectrum of Jupiter, I Geophys. Res., 90, 2673, (1985)

7. R.V. Yelle, R.V., B.R. Sandel, D.E. Shemansky, and S. Kumar, Altitude Variation of EUV Emissions and Evidence for Proton Precipitation at Low Latitudes in the Saturnian Atmosphere, I. Geophys. ‥

8. J.T. Clarke et al., Continued Observations of the H Ly $\alpha$ Emission from Uranus, $\mathbf{I}$. Geophvs. Res., 91, 8771, (1986)

9. J.E.P. Connerney, M. Acuna, and N. Ness, The Magnetic Field of Uranus, J. Geophys. Res., 92, 15329, (1987)

10. R.V. Yelle, J.C. McConnell, B.R. Sandel, and A.L. Broadfoot, The Dependence of Electroglow on the Solar Flux, I. Geophys. Res., 92, 15110, (1987)

11. R. Prange, New Evidence for the Role of Photoelectrons in the $\mathrm{H}_{2}$-Dayglow of the Giant Planets, Astron. Astrphvs., 161, L1, (1986)

12. J.T. Clarke, M.K. Hudson, and Y.L. Yung, The Excitation of the Far UV Electroglow Emissions on Uranus, Saturn, and Jupiter, I. Geophys. Res., 92, 15139, (1987)

13. H. Rishbeth and O.K. Garriott, Introduction to Ionospheric Physics, Academic Press, New York, 1969

14. A. Tan, Spatial and Diurnal Features of the Jovian Equatorial Anomaly, Planet. Space Scj., 34, 117, (1986)

15. H. Rishbeth, The F-Layer Dynamo, Planet. Space Sci., 19, 263, (1971)

16. T.E. Cravens, G.A. Victor, and A. Dalgarno, The Absorption of Energetic Electrons by Molecular Hydrogen Gas, Planet Space Sci., 23, 1059, (1975)

17. A. Dalgarno, M.B. McElroy, and A.I. Stewart, Electron Impact Excitation of the Dayglow, 2. Atmos. Sci., 26, 753, (1969)

18. S. Trajmar, D.F. Register, and A. Chutjian, Electron Scattering by Molecules II. Experimental Methods and Data, Phrs Reports, 27, 219, (1983) 
19. J. Franck and G. Hertz, Verh. der Phvs. Ges., 16, 457, (1914) and 16, 512, (1914)

20. M.K. Hudson, J.T. Clarke, and J. Warren, A Dynamo Theory of Electroglow Emissions from the Outer Planets, submitted to I. Geophys. Res., (1988)

21. B.A. Smith et al., Voyager 2 in the Uranus System: Imaging Science Results, Science, 233, 43, (1986) 